\title{
Parallel Implementation of Runge-Kutta Integrators with Low Storage Requirements
}

\author{
Matthias Korch and Thomas Rauber \\ University of Bayreuth, Department of Computer Science \\ $\{$ korch, rauber\}@uni-bayreuth.de
}

\begin{abstract}
This paper considers the parallel solution of large systems of ordinary differential equations (ODEs) which possess a special access pattern by explicit Runge-Kutta (RK) methods. Such systems may arise, for example, from the semi-discretization of partial differential equations (PDEs). We propose an implementation strategy based on a pipelined processing of the stages of the RK method that does not impose restrictions on the choice of coefficients of the RK method. This approach can be implemented with low storage while still allowing efficient step control by embedded solutions.
\end{abstract}

\section{Introduction}

We consider the parallel solution of initial value problems (IVPs) of ordinary differential equations defined by

$$
\mathbf{y}^{\prime}(t)=\mathbf{f}(t, \mathbf{y}(t)), \quad \mathbf{y}\left(t_{0}\right)=\mathbf{y}_{0}, \quad \mathbf{y}: \mathbb{R} \rightarrow \mathbb{R}^{n}, \quad \mathbf{f}: \mathbb{R} \times \mathbb{R}^{n} \rightarrow \mathbb{R}^{n} .
$$

Parallel numerical solution methods for such problems have been addressed by many authors. An overview is presented in 1]. In this paper, we focus on lowstorage implementations of RK methods suitable for large systems of ODEs where storage space may be the determining factor in the choice of method and implementation variant.

Classical explicit $s$-stage RK methods with method coefficients $a_{l i}, c_{i}$, and $b_{l}$ compute, at each stage $l=1, \ldots, s$, starting with the approximation value $\boldsymbol{\eta}_{\kappa} \approx \mathbf{y}\left(t_{\kappa}\right)$, an argument vector

$$
\mathbf{w}_{l}=\boldsymbol{\eta}_{\kappa}+h_{\kappa} \sum_{i=1}^{l-1} a_{l i} \mathbf{v}_{i}
$$

that is used as input for the function evaluation $\mathbf{f}$ to compute a stage vector

$$
\mathbf{v}_{l}=\mathbf{f}\left(t_{\kappa}+c_{l} h_{\kappa}, \mathbf{w}_{l}\right) .
$$

Finally, the new approximation $\boldsymbol{\eta}_{\kappa+1} \approx \mathbf{y}\left(t_{\kappa+1}\right)$ is computed by:

$$
\boldsymbol{\eta}_{\kappa+1}=\boldsymbol{\eta}_{\kappa}+h_{\kappa} \sum_{l=1}^{s} b_{l} \mathbf{v}_{l}
$$


If the RK method provides an embedded solution, a second approximation $\hat{\boldsymbol{\eta}}_{\kappa+1}$ can be computed using different weights $\hat{b}_{l}$. This second approximation allows an inexpensive estimation of the local error and, hence, an efficient stepsize control.

In the general case, where no assumptions about the method coefficients or the access pattern of the function evaluation can be made, an implementation of the classical RK scheme needs to hold at least $s+1$ vectors of size $n$ (so called registers) to store $\boldsymbol{\eta}_{\kappa}, \mathbf{w}_{2}, \ldots, \mathbf{w}_{s}$, and $\boldsymbol{\eta}_{\kappa+1}$, where $n$ is the dimension of the ODE system. One additional register $\left(\hat{\boldsymbol{\eta}}_{\kappa+1}\right.$ or an error estimate) is required for the implementation of a stepsize controller which can reject and repeat steps.

On modern computer systems the classical approach can be applied to ODE systems with tens or even hundreds of millions of components, since these systems are equipped with several GB of memory, even if a method of high order with a large number of stages is required. Some ODE problems, however, require an even larger number of components. These are, in particular, ODE systems derived from PDE systems by a spatial discretization using the method of lines. For example, a spatial resolution of $1 \%$ leads to a system with $n_{v} \cdot 10^{6}$ components for a 2-dimensional PDE system and $n_{v} \cdot 10^{9}$ components for a 3 -dimensional PDE system, where $n_{v}$ is the number of dependent variables in the PDE system. Increasing the spatial resolution by a factor of 10 leads to an increase in the number of ODE components by a factor of $10^{2}$ for the 2-dimensional PDE system and by a factor of $10^{3}$ for the 3-dimensional PDE system.

In the following, after a discussion of related approaches to reduce the storage space of RK methods in Section 2, we present a new approach based on a pipelined processing of the stages in Section 3. This pipelining approach is applicable to ODE systems with a special access pattern as it is typical for ODE systems derived by the method of lines. It reduces the storage space to a single register plus $\Theta\left(\frac{1}{2} s^{2} \cdot d(\mathbf{f})\right)$, where $d(\mathbf{f})$ is the access distance of the right-handside function $\mathbf{f}$ (see Section 3.1), while preserving all degrees of freedom in the choice of RK coefficients. Stepsize control based on an embedded solution can be realized with only one additional register. Further, a parallel implementation of the pipelining scheme is possible (Section 3.5), which can efficiently utilize parallel computing resources and may be used to reduce the memory requirements of a single computing node even further. Section 4 compares the amount of memory required by the low-storage pipelining implementation with the amount required by other low-storage RK algorithms and by conventional implementations. An experimental evaluation of runtime and scalability of the new implementation is presented in Section 5. Finally, Section 6] concludes the paper.

\section{Related Work}

A first approach to get along with low storage space is the use of RK methods with a small number of stages. This, however, means abandoning desirable properties of the method, such as a high order. Therefore, several authors, e.g., [2 3 4 56], propose special RK methods that can be implemented with low storage space (e.g., 2 or 3 registers) even though they have a larger number of stages 
and a higher order. This is possible by the construction of special coefficient sets such that, at each stage, only the data stored in the available registers is accessed, and the values computed at this stage fit in the available registers. These approaches, however, retain some deficiencies:

1. The coefficient sets must conform to additional constraints. Hence, fewer degrees of freedom are available in the construction of the coefficients. As the result, the methods proposed have weaker numerical properties than classical RK schemes with the same number of stages. If stronger numerical properties are required, one must resort to classical RK schemes with higher memory usage.

2. Using a higher number of stages to reach a specific order leads to higher computational costs and, as a consequence, to a higher runtime.

3. Many of the low-storage RK methods proposed do not provide embedded solutions. Therefore, these methods are suitable only for fixed-stepsize integration, unless additional computations and storage space are invested into stepsize control (e.g., using Richardson extrapolation).

4. Low-storage RK methods with embedded solutions impose additional constraints on the method coefficients [7, thus further reducing the degrees of freedom in the construction of the coefficients.

5. Stepsize control requires additional registers: One additional register is needed to estimate the local error $\left(\hat{\boldsymbol{\eta}}_{\kappa+1}\right.$ or, alternatively, $\left.\mathbf{e}=\hat{\boldsymbol{\eta}}_{\kappa+1}-\boldsymbol{\eta}_{\kappa+1}\right)$. This register may be omitted if the embedded solution $\hat{\boldsymbol{\eta}}_{\kappa+1}$ is computed at stage $s-1$, so that it can be compared with $\boldsymbol{\eta}_{\kappa+1}$, which is computed at stage $s$, without explicitly storing it [7. In order to be able to repeat the time step, a second additional register is needed that saves $\boldsymbol{\eta}_{\kappa}$.

6. Some of the 2-register low-storage RK schemes proposed rely on a specific structure of the coupling between the equations in the ODE system such that, for example, the argument vector of a function evaluation can be overwritten by the result of the function evaluation. If right-hand-side functions with arbitrary access patterns are to be supported, an additional register must be used to store the result of the function evaluation temporarily [2].

It is, therefore, desirable to find new methods or algorithms which overcome these deficiencies. In this paper, we reconsider the pipelining approach suggested, initially, to improve locality and scalability of embedded RK implementations in 89] from this perspective.

\section{Block-Based Pipelining Approach with Reduced Storage Space}

Many sparse ODEs, in particular many discretized PDEs derived by the method of lines, are described by a right-hand-side function $\mathbf{f}=\left(f_{1}, \ldots, f_{n}\right)$ where the components of the argument vector accessed by each component function $f_{j}$ lie within a bounded index range near $j$. In the following we review the pipelining approach proposed in [89], which exploits this property of the ODE system and 
supports arbitrary RK coefficients. We further show how the pipelining approach can be implemented with stepsize control using only ' $2+$ ' registers, i.e., 2 registers plus some small extra space that vanishes asymptotically if the ODE system is very large. Without stepsize control the storage space required even reduces to ' $1+$ ' registers.

\subsection{Access Distance of a Right-Hand-Side Function}

While, in general, the evaluation of one component of the right hand-sidefunction $\mathbf{f}, f_{j}(t, \mathbf{w})$, may access all components of the argument vector $\mathbf{w}$, many ODE problems only require the use of a limited number of components. In many cases, the components accessed are located nearby the index $j$. To measure this property of a function $\mathbf{f}$, we use the access distance $d(\mathbf{f})$ which is the smallest value $b$, such that all component functions $f_{j}(t, \mathbf{w})$ access only the subset $\left\{w_{j-b}, \ldots, w_{j+b}\right\}$ of the components of their argument vector $\mathbf{w}$. We say the access distance of $\mathbf{f}$ is limited if $d(\mathbf{f}) \ll n$.

\subsection{Pipelining Computation Order}

The pipelining computation order described in the following can be applied if the right-hand-side function has a limited access distance. We subdivide all $n$ vectors into $n_{B}=\lceil n / B\rceil$ blocks of size $B$, where $d(\mathbf{f}) \leq B \ll n$. Then, the function evaluation of a block $J \in\left\{1, \ldots, n_{B}\right\}$ defined by

$$
\begin{array}{r}
\mathbf{f}_{J}(t, \mathbf{w})=\left(f_{(J-1) B+1}(t, \mathbf{w}), f_{(J-1) B+2}(t, \mathbf{w}), \ldots,\right. \\
\left.f_{(J-1) B+\min \{B, n-(J-1) B\}}(t, \mathbf{w})\right)
\end{array}
$$

uses only components of the blocks $J-1, J$, and $J+1$ of $\mathbf{w}$ if these blocks exist, i.e., if $1<J<n_{B}$. This dependence structure enables the computation of the blocks of the argument vectors $\mathbf{w}_{2}, \ldots, \mathbf{w}_{s}$, the vector $\Delta \boldsymbol{\eta}=\boldsymbol{\eta}_{\kappa+1}-\boldsymbol{\eta}_{\kappa}$ and the error estimate $\mathbf{e}=\hat{\boldsymbol{\eta}}_{\kappa+1}-\boldsymbol{\eta}_{\kappa+1}$ as illustrated in Fig. 11(a). The computation starts with the first and the second block of $\mathbf{w}_{2}$, which only requires components of $\mathbf{w}_{1}=\boldsymbol{\eta}_{\kappa}$. Then, the first block of $\mathbf{w}_{3}$ can be computed since it only uses components of the first two blocks of $\mathbf{w}_{2}$. In the next step, the third block of $\mathbf{w}_{2}$ is computed which enables the computation of the second block of $\mathbf{w}_{3}$ which again enables the computation of the first block of $\mathbf{w}_{4}$. This is continued until the computation of the first two blocks of $\mathbf{w}_{s}$ has been completed and the first block of $\Delta \boldsymbol{\eta}$ and $\mathbf{e}$ has been computed. Then the next block of $\Delta \boldsymbol{\eta}$ and $\mathbf{e}$ can be determined by computing only one additional block of $\mathbf{w}_{2}, \ldots, \mathbf{w}_{s}$. This computation is repeated until the last block of $\Delta \boldsymbol{\eta}$ and $\mathbf{e}$ has been computed.

\subsection{Working Space of the Pipelining Scheme}

An important advantage of the pipelining approach is that only those blocks of the argument vectors are kept in the cache which are needed for further computations of the current step. One step of the pipelining computation scheme 

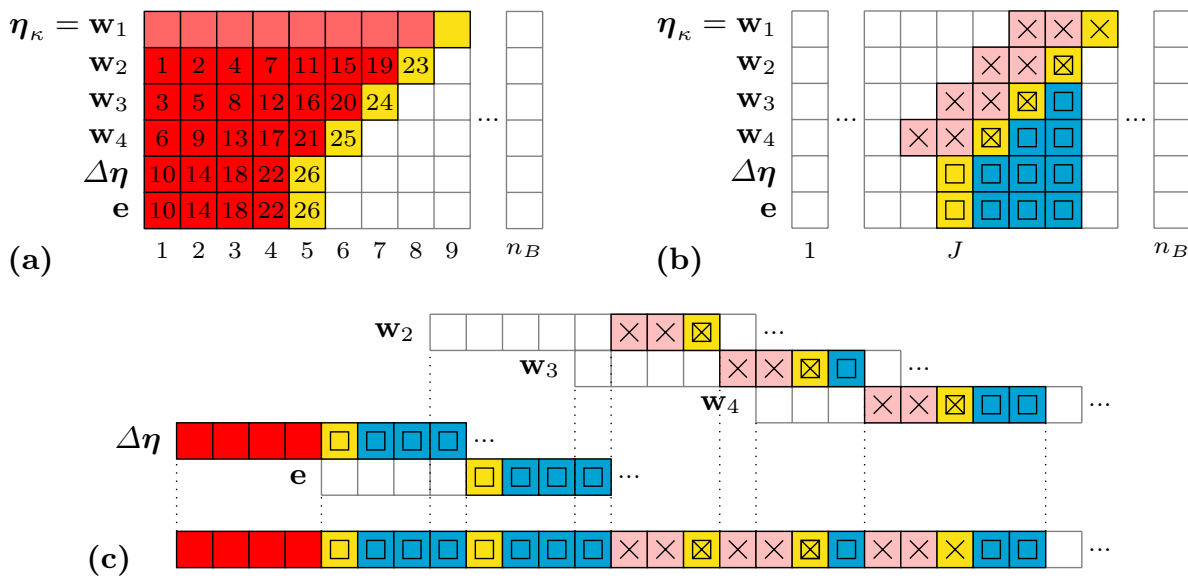

Fig. 1. (a) Illustration of the computation order of the pipelining computation scheme. After the blocks with index 4 of $\Delta \boldsymbol{\eta}$ and e have been computed, the next pipelining step computes the blocks with index 5 of $\Delta \boldsymbol{\eta}$ and e. (b) Illustration of the working space of one pipelining step. Blocks required for function evaluations are marked by crosses. Blocks updated using results of function evaluations are marked by squares. (c) Illustration of the overlapping of the vectors $\Delta \boldsymbol{\eta}$, e and $\mathbf{w}_{2}, \ldots, \mathbf{w}_{s}$ in the lowstorage implementation.

computes $s$ argument blocks and one block of $\Delta \boldsymbol{\eta}$ and $\mathbf{e}$. Since the function evaluation of one block $J$ accesses the blocks $J-1, J$, and $J+1$ of the corresponding argument vector, $3 s$ argument vector blocks must be accessed to compute one block of $\Delta \boldsymbol{\eta}$ and e. Additionally,

$$
\sum_{i=3}^{s}(i-2)=\frac{1}{2}(s-1)(s-2)=\frac{1}{2} s^{2}-\frac{3}{2} s+1
$$

blocks of argument vectors, $s$ blocks of $\Delta \boldsymbol{\eta}$ and $s$ blocks of $\mathbf{e}$ must be updated. Altogether, the working space of one pipelining step consists of

$$
\frac{1}{2} s^{2}+\frac{7}{2} s+1=\Theta\left(\frac{1}{2} s^{2}\right)
$$

blocks of size $B$, see Fig. 1(b). Since $B \ll n$, this is usually only a small part of the working space of $\Theta(s n)$ adherent to general implementations suitable for arbitrary right-hand-side functions which iterate over all $s$ argument vectors in the outermost loop.

\subsection{Low-Storage Implementation of the Pipelining Scheme}

Reconsidering the pipelining computation scheme in terms of storage space, we observe that the pipelining computation scheme, by performing a diagonal sweep across the argument vectors, delivers the blocks of elements of $\Delta \boldsymbol{\eta}$ and $\mathbf{e}$ one after 
the other as the sweep proceeds along the system dimension. The computation of the next blocks of these vectors depends only on those argument vector blocks that belong to the working space of the corresponding pipelining step. Argument vector blocks behind the sweep line that is determined by the working space of the current pipelining step are no longer required for computations in the current time step. Argument vector blocks ahead of the sweep line do not yet contain data computed in the current time step and do not contribute to the computations of the current pipelining step. Hence, a significant reduction of the storage space could be achieved if only those argument vector blocks were kept in memory which are part of the working space of the current pipelining step.

A second opportunity to save storage space offers itself in the computation of the error estimate $\mathbf{e}$. The error estimate $\mathbf{e}$ is used in the decision whether the current step size is to be accepted or whether it is to be rejected and should be repeated with a smaller stepsize. Most step control algorithms make this decision based on a scalar value that is an aggregate of the elements of e, e.g., a vector norm. A common choice for the aggregate $(\epsilon)$ is the maximum norm of e/s divided by some user defined tolerance TOL:

$$
\epsilon=\frac{1}{\mathrm{TOL}}\left\|\frac{\mathbf{e}}{\mathbf{s}}\right\|_{\infty}=\frac{1}{\mathrm{TOL}} \max _{j=1, \ldots, n}\left|\frac{e_{j}}{s_{j}}\right|,
$$

where $\mathbf{s}$ is a vector of scaling factors, e.g., $s_{j}=\max \left\{\left|\eta_{\kappa, j}\right|,\left|\eta_{\kappa+1, j}\right|\right\}$ for $j=$ $1, \ldots, n$. But the computation of aggregates of this kind does not require all elements of e to be stored in memory. Rather, a sweep approach can be applied which updates a scalar variable as the elements of e are computed one after the other: 1

$$
\text { 1.) } \epsilon:=0, \text { 2.) } \epsilon:=\max \left\{\epsilon,\left|\frac{e_{j}}{s_{j}}\right|\right\} \text { for } j:=1, \ldots, n, \quad \text { 3.) } \epsilon:=\frac{\epsilon}{\mathrm{TOL}} .
$$

This sweep approach to compute $\epsilon$ can easily be integrated into the pipelining scheme, which delivers one new block of e after each pipelining step.

All in all, it is sufficient to store the two $n$-vectors $\boldsymbol{\eta}_{\kappa}$ and $\Delta \boldsymbol{\eta}$, the $\frac{1}{2} s^{2}+\frac{3}{2} s-2$ blocks of $\mathbf{w}_{2}, \ldots, \mathbf{w}_{s}$ belonging to the working space of the current pipelining step and $s$ blocks of the error estimate e. This leads to a total amount of

$$
2 n+\left(\frac{1}{2} s^{2}+\frac{5}{2} s-2\right) B=2 n+\Theta\left(\frac{1}{2} s^{2} B\right)
$$

vector elements that have to be stored in memory. If no step control is used, the memory space required even reduces to

$$
n+\left(\frac{1}{2} s^{2}+\frac{3}{2} s-2\right) B=n+\Theta\left(\frac{1}{2} s^{2} B\right)
$$

vector elements, since no elements of $\Delta \boldsymbol{\eta}$ and $\mathbf{e}$ have to be stored.

${ }^{1}$ A similar sweep approach is possible for other $L^{p}$ norms $\|x\|_{p}=\left(\sum_{j=1}^{n}\left|x_{j}\right|^{p}\right)^{\frac{1}{p}}$. 
Table 1. Offsets (in number of blocks) of the overlapping of $\Delta \boldsymbol{\eta}$ with $\mathbf{e}$ and $\mathbf{w}_{2}, \ldots, \mathbf{w}_{s}$

\begin{tabular}{lccccccc}
\hline Offset: & 0 & $s$ & $s+3$ & $\ldots$ & $s+\frac{1}{2} i^{2}+\frac{3}{2} i-2$ & $\ldots$ & $\frac{1}{2} s^{2}+\frac{5}{2} s-2$ \\
Vector: & $\Delta \boldsymbol{\eta}$ & $\mathbf{e}$ & $\mathbf{w}_{2}$ & $\ldots$ & $\mathbf{w}_{i}$ & $\ldots$ & $\mathbf{w}_{s}$ \\
\hline
\end{tabular}

One possibility to design an implementation of a low-storage pipelining scheme which supports stepsize control by an embedded solution is the overlapping of $\Delta \boldsymbol{\eta}$ with the vectors $\mathbf{e}$ and $\mathbf{w}_{2}, \ldots, \mathbf{w}_{s}$ as illustrated in Fig. 1](c). The implementation requires two registers, one of size $n$ which stores the current approximation vector $\boldsymbol{\eta}_{\kappa}$, and one of size $n+\left(\frac{1}{2} s^{2}+\frac{5}{2} s-2\right) B$ which stores $\Delta \boldsymbol{\eta}$ and the elements of e and $\mathbf{w}_{2}, \ldots, \mathbf{w}_{s}$ which belong to the working space of the current pipelining step using the offsets shown in Table 1] Using this overlapping the pipelining scheme can be executed without further changes as described in Section 3.2 . As the result, the working space of the pipelining steps is embedded into the enlarged register holding $\Delta \boldsymbol{\eta}$ as a compact, consecutive window such that the computation of the next block of $\Delta \boldsymbol{\eta}$ moves this window one block forward.

\subsection{Parallel Implementation}

The pipelining scheme can exploit parallelism across the ODE system efficiently by making use of the limited access distance of the ODE system to reduce communication costs and to increase the locality of memory references [8,9. A parallel low-storage implementation of the pipelining scheme with even higher scalability (cf. Section 5) can easily be obtained with only a few adaptations.

We start with a blockwise data distribution of the $n_{B}=\lceil n / B\rceil$ blocks such that every processor is responsible for the computation of $\left\lfloor n_{B} / p\right\rfloor$ or $\left\lceil n_{B} / p\right\rceil$ blocks of $\Delta \boldsymbol{\eta}$ and $\mathbf{e}$, where $p$ is the number of processors. Because in the lowstorage implementation the working space of the pipelining steps is embedded into the register holding $\Delta \boldsymbol{\eta}$, it is not possible to use only one global copy of this register to which all participating processors have shared access. Instead, each processor allocates a chunk of memory of size $\left(\frac{1}{2} s^{2}+\frac{5}{2} s-1\right) B$ locally. The local allocation can be of advantage on shared-memory systems with nonuniform memory access times (NUMA systems) and enables an implementation for distributed address space. It can, however, be a disadvantage on sharedmemory systems with uniform memory access times (UMA systems), because blocks which are required by neighboring processors have to be copied between the local data structures of the threads.

Since the computation of a block $J$ of one argument vector requires the blocks $J-1, J$, and $J+1$ of the preceding argument vector, the computation of the first and the last block of a processor's part of an argument vector requires one block of the preceding argument vector that is stored on a neighboring processor. Therefore, communication between neighboring processors is required during the initialization and the finalization of the local pipelines of the processors. In the parallel low-storage implementation, we use a computation order 
Table 2. Comparison of data set sizes of different RK integrators occurring in the solution of an ODE system of size $n=2 N^{2}$ with access distance $d(\mathbf{f})=2 N$. See explanation in the text.

\begin{tabular}{lcrrrrrr}
\hline & & $\mathrm{I}$ & $\mathrm{II}$ & $\mathrm{III}$ & $\mathrm{IV}$ & $\mathrm{V}$ & \multicolumn{1}{l}{$\mathrm{VI}$} \\
$N$ & $n$ & $S\left(P_{7}^{2 N}\right)$ & \multicolumn{1}{c}{$S(n)$} & $S\left(n+P_{7}^{2 N}\right)$ & $S(2 n)$ & $S\left(2 n+P_{7}^{2 N}\right)$ & $S((7+2) n)$ \\
\hline $10^{2}$ & $2 \cdot 10^{4}$ & $62.5 \mathrm{~KB}$ & $156.2 \mathrm{~KB}$ & $218.8 \mathrm{~KB}$ & $312.5 \mathrm{~KB}$ & $375.0 \mathrm{~KB}$ & $1.4 \mathrm{MB}$ \\
$10^{3}$ & $2 \cdot 10^{6}$ & $625.0 \mathrm{~KB}$ & $15.3 \mathrm{MB}$ & $15.9 \mathrm{MB}$ & $30.5 \mathrm{MB}$ & $31.1 \mathrm{MB}$ & $137.3 \mathrm{MB}$ \\
$10^{4}$ & $2 \cdot 10^{8}$ & $6.1 \mathrm{MB}$ & $1.5 \mathrm{~GB}$ & $1.5 \mathrm{~GB}$ & $3.0 \mathrm{~GB}$ & $3.0 \mathrm{~GB}$ & $13.4 \mathrm{~GB}$ \\
$10^{5}$ & $2 \cdot 10^{10}$ & $61.0 \mathrm{MB}$ & $149.0 \mathrm{~GB}$ & $149.1 \mathrm{~GB}$ & $298.0 \mathrm{~GB}$ & $298.1 \mathrm{~GB}$ & $1.3 \mathrm{~TB}$ \\
$10^{6}$ & $2 \cdot 10^{12}$ & $610.4 \mathrm{MB}$ & $14.6 \mathrm{~TB}$ & $14.6 \mathrm{~TB}$ & $29.1 \mathrm{~TB}$ & $29.1 \mathrm{~TB}$ & $131.0 \mathrm{~TB}$ \\
\hline
\end{tabular}

which differs from that of the implementations described in [89, because the embedding of the working space into the register holding $\Delta \boldsymbol{\eta}$ leads to different dependencies. In particular, processors with even processor ID move from lower block indices to higher block indices, while processors with an odd ID move from higher to lower indices. Thus, processors with even ID initialize their pipeline synchronously with their predecessor and finalize their pipeline synchronously with their successor. Similar to the implementations described in [89], the computation pattern used in the initialization and the finalization phase resembles a zipper, and communication can be overlapped with computations.

\section{Comparison of Data Set Sizes of Semi-discretized PDEs for Different RK Integrators}

The spatial discretization of a $d$-dimensional PDE system by the method of lines [10] uses a $d$-dimensional spatial grid. In the following, we assume that the number of grid points in each dimension be equal, and refer to it as $N$. The ODE system which results from this semi-discretization consists of $n_{v} \cdot N^{d}$ equations, where $n_{v}$ is the number of dependent variables in the PDE system. The spatial derivatives at the grid points are approximated using difference operators which use grid points in a bounded environment of the respective grid point considered. Hence, one spatial dimension can be selected for a subdivision of the ODE system into blocks such that each block represents a $N^{d-1}$-dimensional slice of the grid and the ODE system has a limited access distance $d(\mathbf{f})=n_{v} \cdot r \cdot N^{d-1}$, where $r$ is the maximum distance in the selected dimension between the grid point to which the discretization operator is applied and the grid points used by the discretization operator.

Since the working space of the pipelining scheme requires a storage space of only $\Theta\left(\frac{1}{2} s^{2} d(\mathbf{f})\right)=\Theta\left(n_{v} \cdot r \cdot N^{d-1}\right)$ while the system size $n$ grows with $\Theta\left(N^{d}\right)$, the additional storage space needed to store the working space becomes less significant when $N$ increases. As an example, Table 2 shows a comparison of data set sizes for an ODE of size $n=2 N^{2}$ with access distance $d(\mathbf{f})=2 N$ such as it may result from a 2D PDE with two dependent variables discretized 
using a five-point stencil (e.g., BRUSS2D [1], see also Section 5). The function $S(x)$ used in this table represents the space needed to store $x$ vector elements using double precision ( 8 bytes per vector element). $P_{s}^{B}$ is the number of vector elements in the working space of the pipelining scheme that have to be stored in addition to $\boldsymbol{\eta}_{\kappa}$ (and $\left.\Delta \boldsymbol{\eta}\right)$ if an $s$-stage method and blocksize $B$ is used, i.e.,

$$
P_{s}^{B}=\left(\frac{1}{2} s^{2}+\frac{5}{2} s-2\right) B .
$$

Column I shows the size of the working space $S\left(P_{s}^{B}\right)$ in the case that a 7-stage method is used. We chose $s=7$ because the popular embedded RK method DOPRI5(4) that we used in the runtime experiments presented in Section 5 has 7 stages. Column II shows the size of a single register holding $n$ vector elements. Columns III and V represent the total amount of memory needed when using the low-storage pipelining implementation with (V) and without (III) stepsize control. In comparison to this, column IV shows the amount of memory needed when using the most space-efficient RK methods that have been proposed in related works, which make use of special coefficient sets and require at least two $n$-registers. Finally, column VI shows the storage space required when using a conventional embedded RK method with 7 stages and stepsize control or a conventional RK method with 8 stages without stepsize control.

\section{Experimental Evaluation}

Runtime experiments to evaluate the performance of a sequential and a parallel shared-memory low-storage implementation of the pipelining scheme (PipeDls) in comparison to the pipelining implementation presented in 86] (PipeD) and a conventional RK implementation supporting arbitrary right-hand-side functions (D) have been performed on two off-the-shelf SMP servers equipped with Intel Xeon and AMD Opteron quad-core processors and on two of the largest German supercomputer systems, the Jülich Multiprocessor (JUMP) at the Jülich Supercomputing Centre and the High End System in Bavaria 2 (HLRB 2) at the LRZ Munich. JUMP is a cluster system consisting of 14 SMP nodes, each equipped with 32 Power 6 processors which support simultaneous multithreading (SMT). HLRB 2 is an SGI Altix 4700 system. Currently, the system is equipped with 9728 Intel Itanium 2 Montecito processors at $1.6 \mathrm{GHz}$. The implementations have been developed using $\mathrm{C}$ and POSIX Threads for parallelization. They were compiled with GCC 4.3 on the two $4 \times 4$ SMP servers and on HLRB 2 and with IBM XL C/C++ V 10.1 on JUMP.

As an example problem we use BRUSS2D [1], a typical example of a PDE discretized by the method of lines. Using an interleaving of the two dependent variables resulting in a mixed-row oriented ordering of the components (BRUSS2DMIX, cf. 899), this problem has a limited access distance of $d(\mathbf{f})=2 N$, where the system size is $n=2 N^{2}$. The experiments presented in the following have been performed using the 7-stage embedded RK method DOPRI5(4) and $\alpha=2 \cdot 10^{-3}$ as weight of the diffusion term of BRUSS2D. 


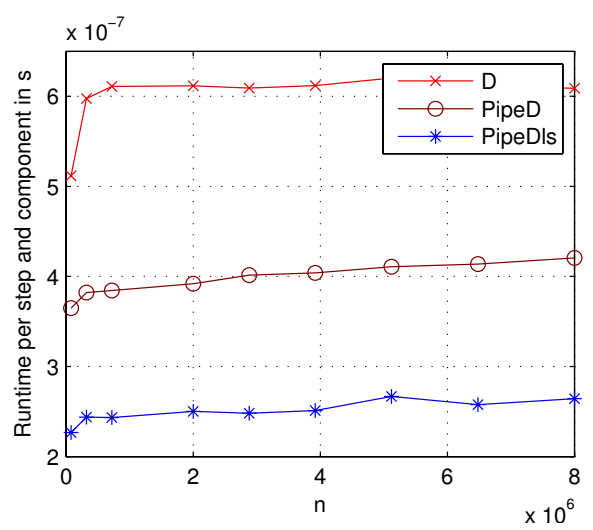

(a) $4 \times 4$ Xeon E7330, $2.4 \mathrm{GHz}$

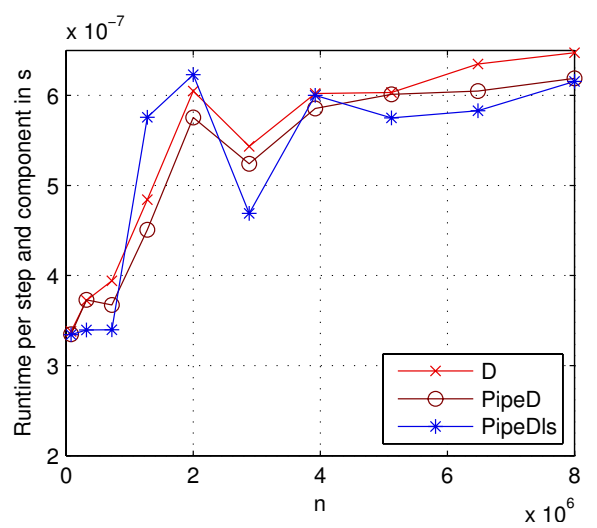

(c) JUMP (Power 6, 4.7 GHz)

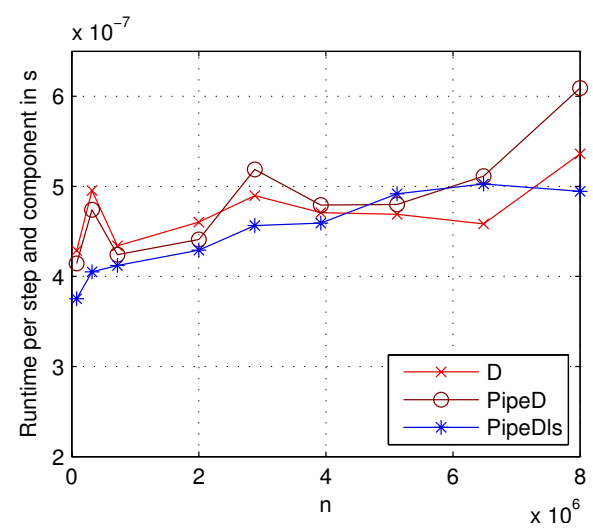

(b) $4 \times 4$ Opteron $8350,2 \mathrm{GHz}$

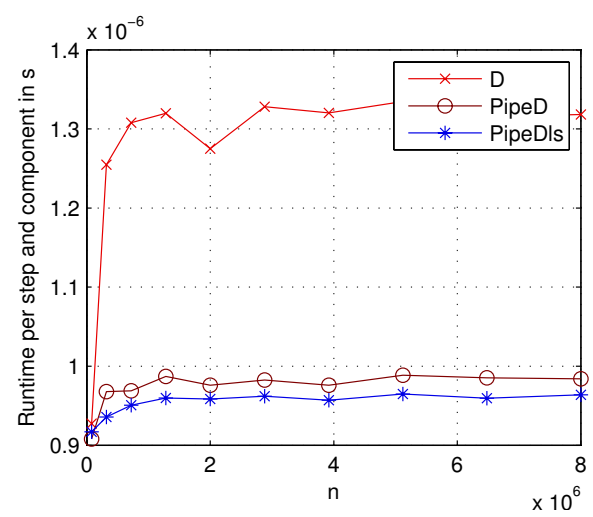

(d) HLRB 2 (Itanium 2 Montecito, $1.6 \mathrm{GHz})$

Fig. 2. Sequential execution time per step and per component against system size $n$

Figure 2 shows a comparison of the sequential runtimes of the 3 implementations on the 4 target systems. On the Opteron processor and on the Power 6 processor the runtimes of the implementations do not differ significantly. But on the Itanium 2 processor the pipelining implementations are considerably faster than the conventional implementation. Moreover, due to its higher locality resulting from the more compact storage of the working space, the low-storage variant of the pipelining scheme runs slightly faster than the implementation presented in 89. The difference in the performance between the two pipelining implementations is even more significant on the Xeon processor.

A comparison of the speedups of the parallel shared-memory implementations on the 4 target systems for selected grid sizes $N$ is shown in Fig. 3, Since our intention is to compare the new low-storage implementation of the pipelining scheme with the pre-existing pipelining implementation from [8], we use the 


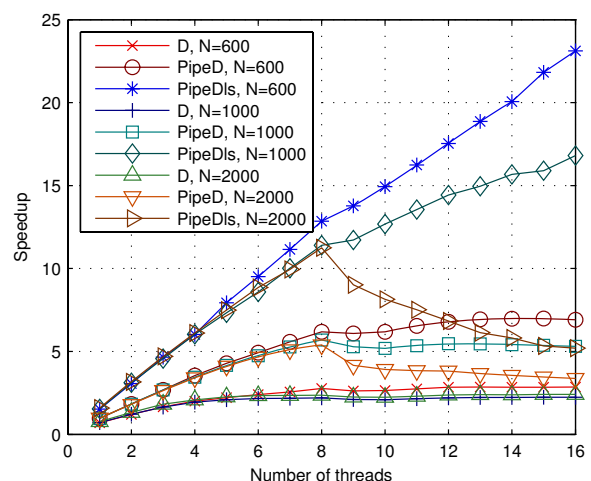

(a) $4 \times 4$ Xeon E7330, $2.4 \mathrm{GHz}$

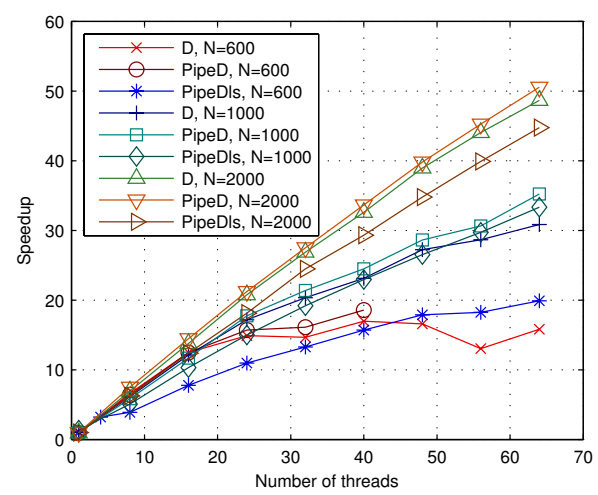

(c) JUMP (Power 6, 4.7 GHz)

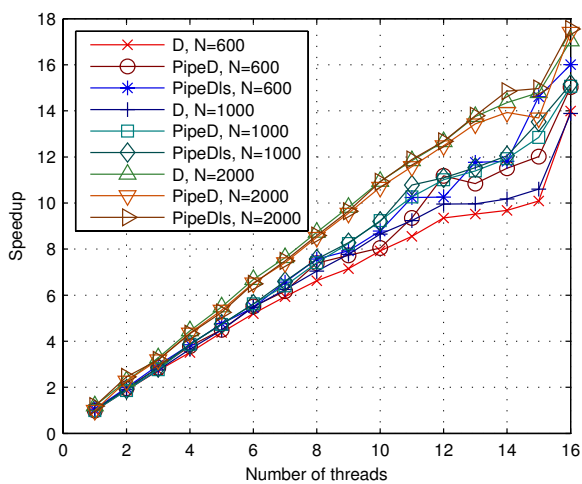

(b) $4 \times 4$ Opteron $8350,2 \mathrm{GHz}$

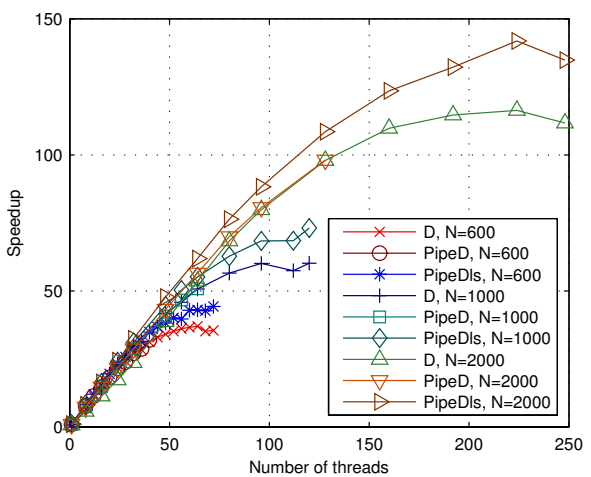

(d) HLRB 2 (Itanium 2 Montecito, $1.6 \mathrm{GHz})$

Fig. 3. Speedup against number of threads for selected grid sizes $N$

sequential execution time of the pre-existing pipelining implementation as the reference for the speedup computation. All 3 implementations scale well for all grid sizes on the 16-core Opteron SMP, where speedups between 14 and 16 have been observed. On JUMP the performance of our current low-storage pipelining implementation is slightly worse than that of the pipelining implementation from 869. We ascribe this to the relatively uniform memory access times on this machine in combination with the fact that the data exchange between neighboring processors is not overlapped by computations. It is remarkable, however, that in most experiments the implementations can take high profit of the SMT capability of the Power 6 processor. On the HLRB 2, where memory access times are substantially more non-uniform, the two pipelining implementations show nearly the same performance. On the Xeon SMP the pipelining implementation from [8] clearly outperforms the general implementation, but its maximum speedup still is below 7 . The low-storage variant of the pipelining scheme reaches significantly higher speedups between 11 and 23 . 


\section{Conclusions}

In this paper, we have proposed an implementation strategy for RK methods which is based on a pipelined processing of the stages of the method. This implementation strategy is suitable for ODE systems with limited access distance. It has been derived from the pipelining scheme presented in 889] by an overlapping of vectors. The implementation strategy proposed herein provides a higher locality of memory references and requires less storage space than the implementations presented in 89 and other implementations proposed in related works. If no step control is required, only $n+\Theta\left(\frac{1}{2} s^{2} d(\mathbf{f})\right)$ vector elements need to be stored. Efficient step control based on an embedded solution is possible using only one additional $n$-vector. In contrast to low-storage RK algorithms proposed by other authors, the presented implementation strategy does not impose restrictions on the choice of coefficients of the RK method and can thus be used with popular embedded RK method such as the methods of Dormand and Prince, Verner, Fehlberg and others. Moreover, a parallel implementation of the proposed strategy is possible which has shown a high scalability on four different parallel computer systems.

Acknowledgments. We thank the Jülich Supercomputing Centre and the LRZ Munich for providing access to their supercomputer systems.

\section{References}

1. Burrage, K.: Parallel and Sequential Methods for Ordinary Differential Equations. Oxford University Press, New York (1995)

2. Kennedy, C.A., Carpenter, M.H., Lewis, R.M.: Low-storage, explicit Runge-Kutta schemes for the compressible Navier-Stokes equations. Appl. Numer. Math. 35(3), 177-219 (2000)

3. Calvo, M., Franco, J.M., Rández, L.: Short note: A new minimum storage RungeKutta scheme for computational acoustics. J. Comp. Phys. 201(1), 1-12 (2004)

4. Berland, J., Bogey, C., Bailly, C.: Optimized explicit schemes: matching and boundary schemes, and 4th-order Runge-Kutta algorithm. In: 10th AIAA/CEAS Aeroacoustics Conference, pp. 1-34 (2004), AIAA Paper 2004-2814

5. Berland, J., Bogey, C., Bailly, C.: Low-dissipation and low-dispersion fourth-order Runge-Kutta algorithm. Computers \& Fluids 35(10), 1459-1463 (2006)

6. Ruuth, S.J.: Global optimization of explicit strong-stability-preserving RungeKutta methods. Math. Comp. 75(253), 183-207 (2005)

7. Kennedy, C.A., Carpenter, M.H.: Third-order $2 N$-storage Runge-Kutta schemes with error control. Technical Report NASA TM-109111, National Aeronautics and Space Administration, Langley Research Center, Hampton, VA (1994)

8. Korch, M., Rauber, T.: Scalable parallel RK solvers for ODEs derived by the method of lines. In: Kosch, H., Böszörményi, L., Hellwagner, H. (eds.) Euro-Par 2003. LNCS, vol. 2790, pp. 830-839. Springer, Heidelberg (2003)

9. Korch, M., Rauber, T.: Optimizing locality and scalability of embedded RungeKutta solvers using block-based pipelining. J. Par. Distr. Comp. 66(3), 444-468 (2006)

10. Schiesser, W.E.: The Numerical Method of Lines. Academic Press Inc., London (1991) 